\title{
Foreword: chromosomes and microtubules-the dynamic duo of mitosis
}

\author{
James G. Wakefield
}

Published online: 8 April 2011

(C) Springer Science+Business Media B.V. 2011

Keywords microtubule $\cdot$ chromosomes $\cdot$ kinetochore $\cdot$ mitotic spindle $\cdot$ mitosis $\cdot$ cell division

$\begin{array}{ll}\text { Abbreviations } \\ \text { aMTOC } & \text { Acentriolar microtubule organizing centre } \\ \text { CPC } & \text { Chromosomal passenger complex } \\ \text { FRET } & \text { Fluorescence resonance energy transfer } \\ \text { GFP } & \text { Green fluorescent protein } \\ \text { HAUS } & \text { Homologous to augmin subunits } \\ \text { HU } & \text { Hydroxyurea } \\ \text { kMT } & \text { Kinetochore microtubule } \\ \text { MT } & \text { Microtubule } \\ \text { MAP } & \text { Microtubule-associated protein } \\ \text { MTOC } & \text { Microtubule organizing centre } \\ \text { NEB } & \text { Nuclear envelope breakdown } \\ \text { PCM } & \text { Pericentriolar material } \\ \text { SAF } & \text { Spindle assembly factor } \\ \gamma \text {-TuRC } & \gamma \text {-Tubulin ring complex } \\ \gamma \text {-TuSC } & \gamma \text {-Tubulin small complex }\end{array}$

Responsible Editor: Herbert Macgregor

J. G. Wakefield $(\bowtie)$

Biosciences, College of Life and Environmental Sciences, University of Exeter,

Stocker Road,

Exeter EX4 4QD, UK

e-mail: j.g.wakefield@exeter.ac.uk
"Mitosis is complicated. Really complicated. You just won't believe how vastly, hugely, mind-bogglingly complicated it is. I mean, you may think DNA replication is pretty incomprehensible, but that's just peanuts to mitosis listen......"

(Apologies to Douglas Adams)

For over a century, scientists have applied a keen eye to the process of cell division or mitosis. Even in the absence of advanced techniques, the dance of the chromosomes during this process was carefully documented with the light microscope before the start of the twentieth century. Now, early in the twenty-first century, the molecular changes that occur during this dance are rapidly being identified. A search of the Gene Ontology Database lists 321 human proteins that are involved in mitosis (www.thegeneontology. com). This is a gross underestimate (and says something about the difficulties associated with categorizing protein function) considering that the kinetochore (the structure on each chromatid responsible for chromosome motion) is composed of at least 100 proteins, that there are scores of microtubuleassociated proteins involved in division, and that there are thousands of proteins associated with mitotic chromosomes and the other structures (e.g. the centrosome) involved in mitosis.

This special issue of Chromosome Research contains a series of articles on the two main structural 
elements behind mitosis. They highlight the complexity of this research topic as well as the progress made in understanding how chromosome segregation occurs, while also suggesting new ways to think about some of the elements and processes involved.

We begin with a personal view of mitosis. Scientific research is not limited to experimental advances themselves; it is also an inherently historical and social pursuit. The researchers to whom I was exposed during my $\mathrm{PhD}$ studies in the mid-90s were the Mitchisons, Salmons and Karsentis of the field, to name a few. And, of course, Conly Rieder, whose micrographs showing the changing distribution of the chromosomes and microtubules through mitosis, adorned the pages of that core textbook, Molecular Biology of the Cell. Shortly after this special issue was initiated, Conly spent 6 months with Bill Earnshaw in Edinburgh, after which it was rumoured that he would be 'retiring'. This visit to the UK seemed like the ideal opportunity to interview him about his life at the cutting edge of mitosis research. The edited result of this session is presented after this foreword and, in my opinion, does not disappoint; his candid appraisal of the field, its reliance on observational techniques and its future directions all give pause for thought.

Following his interview Conly discusses the checkpoint pathways that regulate mitotic progression - in a tour de force of personal opinions described by the author as a 'brief review'. It starts with a re-appraisal of when mitosis begins, and then moves onto the metaphase-anaphase transition. In this review, he emphasizes some of the nomenclature and methods that have led to confusion in interpreting experimental results. The author is just as direct when addressing the long-standing question of kinetochore attachment versus tension, concluding that "it is the constant production of unattached kinetochores from syntelic attachments by the error correction mechanism that delays checkpoint satisfaction in taxol-treated cells". After dealing with the dangers of ambiguous terms like "antimitotic agents", "mitotic inhibitors" and "endoreduplication", his final conclusion is that one should always determine directly how individual living cells respond to a protocol before scaling it up for population studies. Since this information can be obtained simply by following live cells via lowmagnification phase-contrast light microscopy, it trans- lates to a view that is perhaps not so different from that proffered by those who documented cell division a century ago.

Leading on from this, Sandra Moser and Jason Swedlow consider chromosome composition by reviewing the main non-histone proteins associated with mitotic chromatin and their functions. Again, the starting point is historical, beginning with the "chromosomal scaffold" conceived in the 1970s from electron microscopy and enzymatic digestion studies. The article then discusses how the biophysical properties of chromosomes have been investigated before documenting recent work from the Earnshaw lab, which has identified several thousand candidate chromosomal proteins (Ohta et al. 2010). The main players in mitotic chromosome structure are then reviewed, including SMCs, topoisomerase II, condensins and cohesins - all the while emphasizing the correlation between advances in understanding mitosis with advances in microscopy techniques, before turning to the histones and the post-translational modifications that drive chromosome compaction. Finally, the article discusses the interface between microtubules and chromosomes, as well as techniques that allow an estimation of the forces applied by and to the microtubules during chromosome motion.

This endpoint leads into the next four articles, which concentrate on the mitotic apparatus or "spindle". Beginning with a brief review of the mechanisms that underlie spindle formation, my colleague Tommy Duncan and I opine that the microtubules of the spindle arise not just from centrosomes and/or chromatin, but also from additional sites including the growing spindle itself (through the augmin complex), the nuclear envelope and spontaneously within the cytoplasm. We then consider why certain mechanisms predominate, focusing on the spatial and temporal restrictions placed upon them. Finally, we provide a broad overview of where the next advances in the field may arisehighlighting insights that can be gained from a holistic approach combining in vitro biochemistry, computational modelling, qualitative observation and approaches involving quantitative image analysis.

The next review by Han Zhang and Kelly Dawe focuses on spindle formation in plants as an example of cells which lack centrosomes. The intricacies of chromatin-driven, Ran-dependent spindle formation 
are considered, as is the functional conservation between the main players-NuMA, TPX2, RanGAP and Rae1. The discussion then turns to sites of microtubule nucleation analogous to the animal centrosome. These include the relationship between histone $\mathrm{H} 1$ and tubulin at the nucleus/cytoplasm interface as well as the pre-prophase band, a ring of cortical microtubules that forms during late $\mathrm{G} 2$ at the (future) division plane. By consistently emphasizing that their perspective is based on sparse plant literature and therefore relies heavily on data from other systems, this review also argues that there is still much that plant spindle formation can tell us about the way microtubules are generally organised during mitosis.

Two different takes on the nature of the spindle follow. In the first, Kristen Johansen and colleagues revisit evidence for a spindle matrix. A discussion of the composition and physical nature of the nuclear pore complex and, specifically, the subset of FG-repeat-containing proteins that possess elastic hydrogel-like properties, leads to the hypothesis that many of these FG-proteins are also components of a malleable, flexible spindle matrix structure, capable of excluding organelles like as mitochondria, but permeable and flexible enough to allow MTs to form the spindle around them. Following this thoughtprovoking section, it is proposed that other classes of molecules including polyADPribose, actin/myosin and membranes (in the form of re-organised ER or nuclear envelope) are also part of the matrix. Finally, the authors discuss the major problem that has hindered acceptance of the matrix as a reality, viz. the lack of evolutionary conservation of many matrix proteins at the level of amino acids. However, by evaluating what is known regarding one of the few conserved matrix proteins, Tpr, there is little doubt that a structure, independent of the microtubulebased spindle apparatus and formed at least partly from nuclear proteins, contributes to chromosome segregation.

The next article, by Mark Alliegro, presents an alternative, but by no means, mutually exclusive picture. In it, the spindle is described as a "ribonucleoprotein complex"- a statement easy to verify biochemically, but whose relevance is much disputed. The provocative introduction, "thinking of the cell division apparatus ....as a comprehensive unit com- posed of all classes of biomolecules (almost a cell within a cell)", sets the scene for the article, which begins by considering several studies from the 1950s that biochemically confirmed the presence of RNA in the mitotic apparatus. After discussing the difficulties in separating contaminants from "real" components, the author reviews the morphological, functional and accessory evidence that nucleic acids are part of the mitotic apparatus. The latter half of the review focuses on the identity of specific nucleic acids in the centrosome and spindle, noting that, while over 200 RNAs have been identified in various organisms, some (e.g. cyclin B mRNA) are well accepted by the field, and others (e.g. potential remnants of symbiogenetic events) are not. We are then treated to a review of the nucleolinus, first described in 1857 (Agassiz 1857) and generally regarded as a distinct part of the nucleolus. The author summarises evidence that the nucleolinus plays a role in meiotic spindle formation in the surf clam, Spisula. Finally, the study tackles the possibility that the presence of RNAs at/in the centrosome and spindle are relevant to the origin and evolution of these structures.

Next, we switch from the spindle to chromosomes with several articles about the kinetochore. The first, by John Tooley and Todd Stukenberg, deals with the hetero-tetrameric kinetochore complex- Ndc80. From its origins as a contaminant in yeast spindle pole body preparations, we are guided through to 8.6 A cryo-electron microscopy structural studies showing that the complex is dumbbell-shaped (Alushin et al. 2010). The authors then cite in vitro experiments as well as their own unpublished work to speculate how Ndc80 functions to use MT shortening to power chromosome motion and how it is regulated by AuroraB and Nek2A kinases. The latter part of the article contains a model for Ndc 80 function. To finish, the authors compare the structure-function relationships of Ndc80 in humans (where it is essential) and yeast (where it is not); re-enforcing the possibility that these differences are due to the presence of another potential MT clamp, the Dam1 (DASH) complex.

Leading directly on, Graham Buttrick and Jonathan Millar introduce DASH as a kinetochore-associated oligomer capable of converting kinetochore microtubule depolymerizing plus ends to force generation. Noting that the complex does not form rings around microtubules in vivo, and drawing on the number of 
DASH complexes estimated by the Salmon lab (Joglekar et al. 2006), they conclude "even if all DASH complexes were clustered onto a single microtubule, there would not be enough to form a single microtubule ring". This leads to the exploration of complementary mechanisms, such as the cooperation between DASH and Ndc80, the phosphoregulation of the complex by Ipl (AuroraB) and Mps1, and the idea that similar functions may be elicited in higher eukaryotes by CENP-E or the newly identified Ska complex. Finally, the authors provide a compelling argument, based on the mechanistic differences between kinetochore-microtubule interactions in cerevisiae, pombe and mammalian cells, as to why a DASH analogue is apparently not required in all eukaryotes.

Next, we have an article from Andrew McAinsh's lab, which views the kinetochore as "a set of interacting springs, clutches and motors", and explores the use of mathematical and statistical models to understand how these units drive kinetochore function. It details the current models that explain the pull and push required to oscillate chromosomes, while not overlooking their limitations such as kinetochore stretching or the influence of neighbouring chromosome arms. After a comprehensive analysis of Civelekoglu-Scholey's force-balance models derived from Drosophila embryos, the authors assess the modelling difficulties posed by sister kinetochores that are not bioriented, noting that although mono-oriented and merotelically attached chromosome movements have been modeled, syntelic attachments (perhaps because they are so rapidly corrected) have not. Finally, the authors define key questions that need to be answered before an integrated model of chromosome oscillations can be constructed. Their conclusion, that such an effort will require improved live $3 \mathrm{D}$ imaging technologies describing kinetochore trajectories and transitions under multiple perturbations, is one that will undoubtedly resonate with most cell biologists.

The penultimate article in this issue, by Uttama Rath and David Sharp, enhances the previous three by asking precisely how MTs are depolymerised to allow chromosomes to move-both at their kinetochore-associated ends and also at the spindle pole (mechanisms termed "Pacman" and "Flux", respectively). After a historical background, the authors turn to the molecular components orchestrating these behaviours-pole-associated depolymerases, severing enzymes and microtubule-sliding motors. They provide data suggesting that Drosophila kinesin-13 depolymerases (Klp10A and Klp59D) cooperate with the spindle pole protein Asp to regulate flux, before moving on to describe the nuanced roles of MT-severing enzymes, Spastin and Fidgitin. The section on flux ends in a discussion on the role of kinesin-5 motors, which localize to the overlapping MTs of the central spindle, and on gaps in our understanding of how they function. Turning their attention to Pacman, which in some mammalian cells accounts for up to $80 \%$ of the chromosome poleward movement, the authors argue that the mechanisms used are strikingly similar to those involved in flux. In all, this mirrored interpretation of plus- and minus-end microtubule dynamics provides a thought-provoking look at what occurs at both ends of a kinetochore microtubule during mitosis.

This special issue of Chromosome Research finishes with a topic that underlies the motivation of many individuals working in the field of cell division - the disregulation of mitosis that leads to transformation and cancer. The article, by Sarah Thompson and Duane Compton, begins by defining the two types of chromosomal abnormalities seen in tumours - the loss or gain of whole chromosomes (Chromosomal Instability, CIN) and structural rearrangements involving multiple chromosomes (Chromosome Structure Instability-CSI), before moving on to describing the mechanisms underlying these phenomena. The authors argue that the cause of CIN is merotely which is not visible to the spindle checkpoint, and they detail the ways in which increases in merotely likely occur. Concerning CSI, they note that although some cancers are driven by a single, specific and recurrent rearrangement, solid tumours often possess cells containing many different and complex chromosomal changes. Next, they turn to the mechanisms that prevent aneuploidy, citing the startling fact that although human cells mis-segregate chromosomes once every 100 divisions, an adult human has about a hundred million cells dividing at any one time. The article ends on the question of why chromosomally abnormal cells are resistance to chemotherapies. The message that comes across - that the disregulated population of cells 'sample the genomic landscape' in order to overcome 
limiting restraints - truly illustrates the complexity underlying chromosome segregation when viewed in the context of a cell and its environment.

Finally, I hope that after reading the articles in this issue of Chromosome Research, you will appreciate the complexities of the mitotic process and the challenges that it presents for future research. I would like to thank all the authors for their excellent contributions and all referees for their valuable suggestions on manuscripts. I wish also to thank Professor Herbert Macgregor, editor-in-chief of Chromosome Research and his editorial board for allowing me the opportunity to assimilate this special issue, and for their enthusiasm and support.

\section{References}

Agassiz L (1857) Contributions to the Natural History of the United States of America. First Monograph. Part IIL Embryology of the Turtle. Boston

Alushin GM, Ramey VH, Pasqualato S, Ball DA, Grigorieff N, Musacchio A, Nogales E (2010) The Ndc80 kinetochore complex forms oligomeric arrays along microtubules. Nature 467:805-810

Joglekar AP, Bouck DC, Molk JN, Bloom KS, Salmon ED (2006) Molecular architecture of a kinetochore-microtubule attachment site. Nat Cell Biol 8:581-585

Ohta S, Bukowski-Wills JC, Sanchez-Pulido L et al (2010) The protein composition of mitotic chromosomes determined using multiclassifier combinatorial proteomics. Cell $142: 810-821$ 